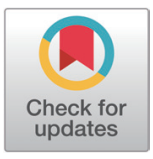

Received: Oct 22, 2020

Revised: Jan 20, 2021

Accepted: Jan 21, 2021

\#These authors contributed equally to this work.

*Corresponding author

Hyeun Bum Kim

Department of Animal Resources

Science, Dankook University, Cheonan

31116 , Korea.

Tel: +82-41-550-3653

E-mail: hbkim@dankook.ac.kr

Ju-Hoon Lee

Department of Food Animal

Biotechnology, Department of

Agricultural Biotechnology, Center

for Food and Bioconvergence, Seoul

National University, Seoul 08826,

Korea.

Tel: +82-2-880-4854

E-mail: juhlee@snu.ac.kr

Copyright $(\subset) 2021$ Korean Society of Animal Sciences and Technology. This is an Open Access article distributed under the terms of the Creative Commons Attribution Non-Commercial License (http:// creativecommons.org/licenses/by$\mathrm{nc} / 4.0 /$ ) which permits unrestricted non-commercial use, distribution, and reproduction in any medium, provided the original work is properly cited.

\section{Complete genome sequence of Escherichia coli K_EC180, a bacterium producing shiga-like toxin isolated from swine feces}

\author{
Hyeri $\mathrm{Kim}^{1 \#}$, Jae Hyoung Cho ${ }^{1 \#}$, Jin Ho Cho ${ }^{2 \#}$, Minho Song ${ }^{3 \#}$, Hakdong Shin ${ }^{4}$, \\ Sheena Kim ${ }^{1}$, Eun Sol Kim ${ }^{1}$, Hyeun Bum Kim ${ }^{1 *}$ and Ju-Hoon Lee ${ }^{5 *}$ \\ ${ }^{1}$ Department of Animal Resources Science, Dankook University, Cheonan 31116, Korea \\ ${ }^{2}$ Division of Food and Animal Science, Chungbuk National University, Cheongju 28644, Korea \\ ${ }^{3}$ Division of Animal and Dairy Science, Chungnam National University, Daejeon 34134, Korea \\ ${ }^{4}$ Department of Food Science and Biotechnology, College of Life Science, Sejong University, Seoul \\ 05006, Korea \\ ${ }^{5}$ Department of Food Animal Biotechnology, Department of Agricultural Biotechnology, Center for Food \\ and Bioconvergence, Seoul National University, Seoul 08826, Korea
}

\begin{abstract}
Escherichia coli normally colonizes the lower intestine of animals and humans, but some serotypes are foodborne pathogens. The Escherichia coli K_EC180 was isolated from swine feces that were collected from a weaner pig. In this genome announcement, E. coli K_EC180 was sequenced using PacBio RS II and Illumina NextSeq 500 platforms. The complete chromosome of $E$. coli K EC180 is composed of one circular chromosome $(5,017,281 \mathrm{bp})$ with $50.4 \%$ of guanine + cytosine $(G+C)$ content, 4,935 of coding sequence (CDS), 88 of tRNA, and 22 of rRNA genes. The complete genome of E. coli K_EC180 contains the toxin genes such as shiga-like toxins (stXA and stxB).
\end{abstract}

Keywords: Escherichia coli K_EC180, Swine feces, Whole genome sequencing, Shiga-like toxin

\section{INTRODUCTION}

Escherichia coli is a facultative anaerobic bacterium which is commonly spread on biosphere. E. coli normally colonizes the lower intestine of animals and humans (1). However, Some of the serotypes such as Enterohemorrhagic E. coli (EHEC), Enterotoxigenic E. coli (ETEC), Enteropathogenic E. coli (EPEC) and Shiga toxin-producing $E$. coli (STEC) can cause foodborne illnesses in people.

E. coli $\mathrm{K} \_$EC180 was isolated from swine feces that were collected from a livestock farm in Haenamgun, Jeollanam-do, Korea. E. coli K_EC180 was streaked to Luria-Bertani (LB) agar and incubated at $37^{\circ} \mathrm{C}$ for $24 \mathrm{~h}$. The suspected colony in LB agar was inoculated into LB broth and incubated at $37^{\circ} \mathrm{C}$ for $24 \mathrm{~h}$. To analyze the complete genome, the E. coli K_EC180 genome was sequenced by PacBio RS II (Pacific Biosciences, Menlo Park, CA, USA) at Insilicogen (Yongin, Korea) and Illumina NextSeq 500 (Illumina, San Diego, CA, USA) platform at LabGenomics (Seongnam, Korea). The genomic DNA of E. coli K_EC180 for PacBio and Illumina sequencing was extracted using the MagAttract 
ORCID

Hyeri Kim

https://orcid.org/0000-0002-6560-2390

Jae Hyoung Cho

https://orcid.org/0000-0002-1128-3451

Jin Ho Cho

https://orcid.org/0000-0001-7151-0778

Minho Song

https://orcid.org/0000-0002-4515-5212

Hakdong Shin

https://orcid.org/0000-0001-7615-9809

Sheena Kim

https://orcid.org/0000-0002-5410-1347

Eun Sol Kim

https://orcid.org/0000-0001-8801-421X

Hyeun Bum Kim

https://orcid.org/0000-0003-1366-6090

Ju-Hoon Lee

https://orcid.org/0000-0003-0405-7621

Competing interests

No potential conflict of interest relevant to

this article was reported.

Funding sources

The present study was supported by the research fund (19162MFDS037) from the

Ministry of Food and Drug Safety, Republic of Korea, and by the University Innovation Support Program through the National Research Foundation of Korea (NRF) funded by the Ministry of Education (Dankook University 2019).

\section{Acknowledgements}

We thank Mo Re Kim (Brandeis University, MA, USA) for the English grammar corrections.

Availability of data and material Upon reasonable request, the datasets of this study can be available from the corresponding author.

\section{Authors' contributions}

Conceptualization: Cho Jin Ho, Song MH, Kim HB, Lee JH.

Data curation: Kim H, Shin H, Kim S, Kim ES.

Formal analysis: Kim H, Shin H, Kim S, Kim ES.

Methodology: Kim H, Cho Jae Hyoung, Song $\mathrm{MH}$

Software: Kim H, Cho Jae Hyoung, Song $\mathrm{MH}$.

Validation: Kim H, Shin H, Kim S, Kim ES.

Investigation: $\mathrm{Kim} \mathrm{H}$, Cho Jin Ho, Song MH, Kim HB, Lee JH.

Writing - original draft: Kim H, Cho Jae Hyoung, Song MH, Kim HB, Lee JH.

Writing - review \& editing: Kim H, Cho Jin $\mathrm{Ho}$, Song MH, Kim HB, Lee JH.

Ethics approval and consent to participate This article does not require IRB/IACUC approval because there are no human and animal participants.
HMW DNA Kit (QIAGEN), and NucleoSpin ${ }^{\circledR}$ Microbial DNA kit (TAKARA) according to the manufacturer's instructions. Library preparation was conducted using SMRTbell ${ }^{\mathrm{TM}}$ Template Prep Kit 1.0 for Pacbio (Pacific Biosciences) and TruSeq DNA Sample Preparation Kit for Illumina (Illumina) according to the manufacturer's instructions. PacBio sequencing yielded 1,131,537,370 base pairs and 145,423 long reads after filtering, and 9,199,306 paired-end reads with 1,389,095,206 bp were obtained with Illumina sequencing. De novo assembly was conducted using the hierarchical genome assembly process (HGAP v2.3.0) workflow (Chin et al., 2013) and polished using Quiver. Subsequently, Illumina NextSeq reads were aligned to the PacBio RSII assembly using Burrows-Wheeler Aligner (BWA)-MEM v0.7.17-r1188, and the errors were corrected by using Pilon version $1.23(2,3)$. The quality of genome assembly and the validaty of the final genome were assessed using Quality Assessment Tool for Genome Assemblies (QUAST) v5.0.2 and Benchmarking Universal Single-Copy Orthologs (BUSCO) v3.0.2 (4, 5). Open reading

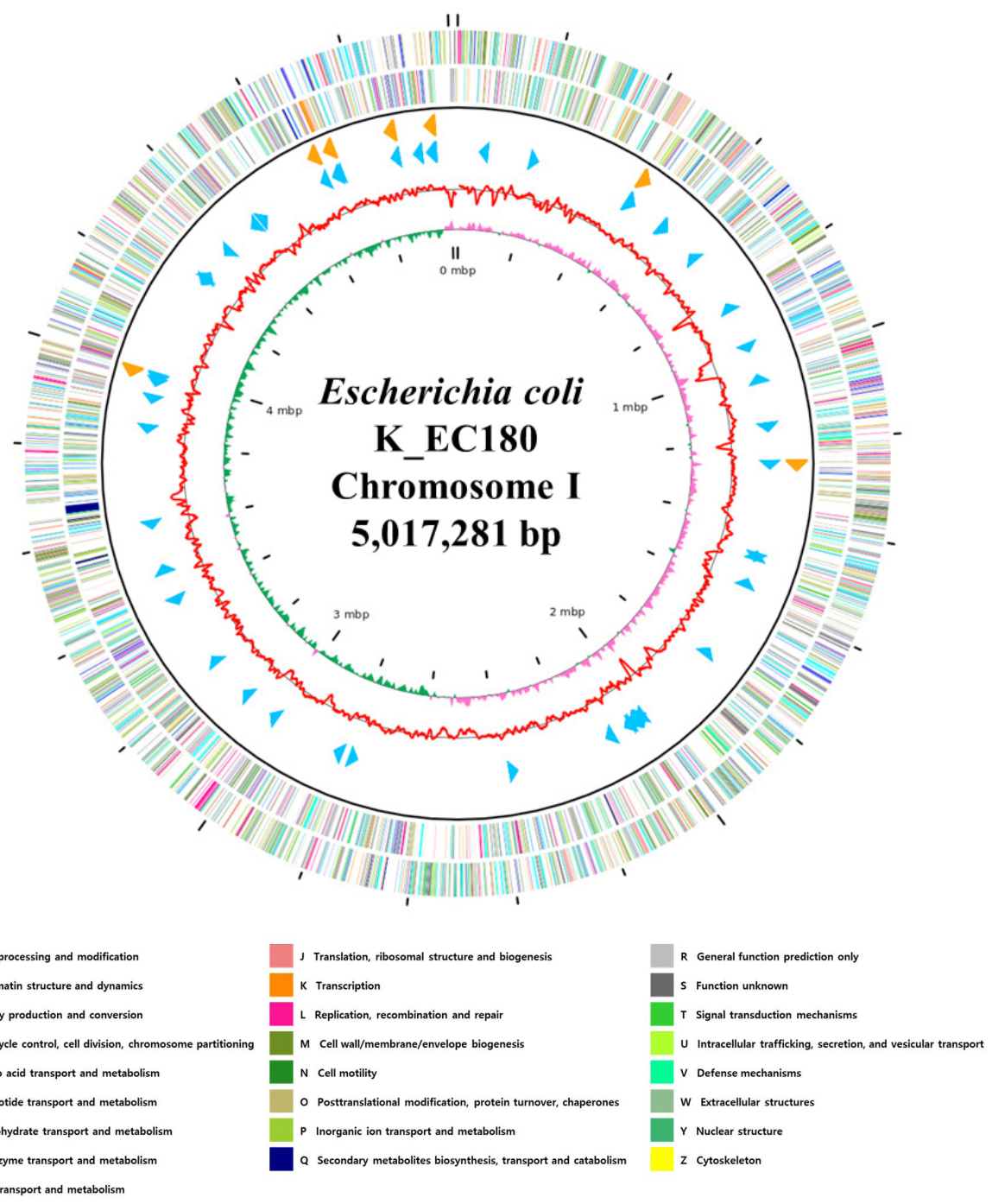

Fig. 1. Genome map of Escherichia coli K EC180. The outer circle denotes the locations of all annotated ORFs, and the inner circle with the red denotes GC content. Pink, and green peaks denote GC skew. The orange arrows denote rRNAs, and the sky blue arrows denote the tRNA operons. All annotated ORFs are colored differently based on the COG assignments. ORFs, open reading frames; G, guanine; C, cytosine; COG, clusters of orthologous groups. 
Table 1. Genome features of Escherichia coli K_EC180

\begin{tabular}{lc}
\hline \multicolumn{1}{c}{ Property } & Term \\
\hline Libraries used & PacBio SMRTbell TM library \\
Sequencing platforms & TruSeq DNA Sample Preparation Kit \\
Assemblers & PacBio RS Il sequencer \\
Illumina NextSeq 500 \\
Annotation method & PacBio SMRT analysis v2.3.0 HGAP.3 \\
Average genome coverage & PROKKA v1.14.5 and RAST v2.0 \\
Chromosome length (bp) & $100 \times$ \\
No. of contigs & $5,017,557$ bp \\
Guanine + cytosine (G + C) content (\%) & 1 \\
Protein-coding genes (CDSs) & 50.4 \\
rRNA genes & 4,935 \\
tRNA genes & 22 \\
Plasmids & 88 \\
Genbank Accession No. & 0 \\
\hline
\end{tabular}

frames (ORFs) and RNA genes of E. coli K_EC180 were predicted and functionally annotated through rapid prokaryotic genome annotation (PROKKA) v1.14.5 (6) and Rapid Annotation using Subsystem Technology (RAST) v2.0 (7). The functional categorization and classification of all predicted ORFs were conducted using the RAST server-based SEED viewer and Clusters of Orthologous Groups (COG) - based EggNOG. The putative virulence factors and Antimicrobial resistance were described using BLAST according to the Virulence Factor Database (VFDB) (8). The whole genome of $E$. coli K_EC180 is composed of one circular chromosome $(5,017,281 \mathrm{bp})$ with $50.4 \%$ of $\mathrm{G}+\mathrm{C}$ content, 4,935 of coding sequence (CDS), 88 of tRNA, and 22 of rRNA genes.

The complete genome of $E$. coli K_EC180 contains the toxin genes encoding shiga-like toxin (stx2e subunit $\mathrm{A}$ and stx2e subunit B), which may cause diseases in humans by damaging small blood vessels in places such as the digestive tract, kidneys and central nervous system $(9,10)$. E. coli $\mathrm{K}-\mathrm{EC} 180$ also possessed ess $C$, esc $V$, esc $R$, esc $S$, esc $V$, and esc J genes which involved in a type III secretion system. In addition, there were fim (A to $\mathrm{H}$ ) genes encoding Type I fimbriae. We summarized the general properties of the E. coli K_EC180's complete genome in the Fig. 1 and Table 1.

\section{DATA AVAILABILITY}

The complete genome sequences of E. coli $K \_$EC180 were deposited in GeneBank under the accession numbers CP062203. The BioSample accession number is SAMN16277032, and BioProject accession number is PRJNA666028.

\section{REFERENCES}

1. Guevarra RB, Lee JH, Lee SH, Seok MJ, Kim DW, Kang BN, et al. Piglet gut microbial shifts early in life: causes and effects. J Anim Sci Biotechnol. 2019;10:1. https://doi.org/10.1186/ s40104-018-0308-3

2. Li H. Aligning sequence reads, clone sequences and assembly contigs with BWA-MEM. arXiv. https://arxiv.org/abs/1303.3997 
3. Walker BJ, Abeel T, Shea T, Priest M, Abouelliel A, Sakthikumar S, et al. Pilon: an integrated tool for comprehensive microbial variant detection and genome assembly improvement. PLOS ONE. 2014;9:e112963. https://doi.org/10.1371/journal.pone.0112963

4. Simão FA, Waterhouse RM, Ioannidis P, Kriventseva EV, Zdobnov EM. BUSCO: assessing genome assembly and annotation completeness with single-copy orthologs. Bioinformatics. 2015;31:3210-2. https://doi.org/10.1093/bioinformatics/btv351

5. Gurevich A, Saveliev V, Vyahhi N, Tesler G. QUAST: quality assessment tool for genome assemblies. Bioinformatics. 2013;29:1072-5. https://doi.org/10.1093/bioinformatics/btt086

6. Seemann T. Prokka: rapid prokaryotic genome annotation. Bioinformatics. 2014;30:2068-9. https://doi.org/10.1093/bioinformatics/btu153

7. Aziz RK, Bartels D, Best AA, DeJongh M, Disz T, Edwards RA, et al. The RAST server: rapid annotations using subsystems technology. BMC Genomics. 2008;9:75. https://doi. org/10.1186/1471-2164-9-75

8. Liu B, Zheng D, Jin Q, Chen L, Yang J. VFDB 2019: a comparative pathogenomic platform with an interactive web interface. Nucl Acids Res. 2019;47:D687-92.

9. Tesh VL, O'Brien AD. The pathogenic mechanisms of Shiga toxin and the Shiga-like toxins. Mol Microbiol. 1991;5:1817-22. https://doi.org/10.1111/j.1365-2958.1991.tb00805.x

10. O’Brien AD, Holmes RK. Shiga and Shiga-like toxins. Microbiol Rev. 1987;51:206-20. https://doi.org/10.1128/MR.51.2.206-220.1987 